were given drugs both by continuous subcutaneous infusion and as stat doses. The drugs were most often given by generalist community nurses or nursing home staff (91\%). There was little difference between drug prescription and administration in malignant or non-malignant disease.

Conclusions When prescribe, injectable medication is frequently used in the last week of life, especially diamorphine, midazolam, cyclizine and glycopyrronium. Administration is usually by staff who are not specialist in palliative care, highlighting the need for support and education for community healthcare professionals.

\section{P-43 EVALUATION OF OPIATE PRESCRIBING AND ADJUSTMENT IN RENAL IMPAIRMENT IN AN ACUTE MEDICAL ADMISSIONS UNIT}

${ }^{1}$ Kate Howorth, ${ }^{1}$ Katie Frew, ${ }^{1} J a n e$ Atkinson, 'Eleanor Grogan, 'Alastair Green, ${ }^{1}$ Emma Foggett. ${ }^{1}$ Northumbria Healthcare NHS Foundation Trust, Cramlington, UK

10.1136/bmjspcare-2017-00133.43

Background Incorrect opioid prescribing can have significant consequences for patient safety and quality of care (1), adjustment of opioid is often needed in renal impairment $(2,3)$. The audit aimed to assess if an acute medical assessment unit (AMU) was meeting current guidance regarding opioid prescribing in acute medical admissions

Methodology A retrospective case note audit was conducted of all patients admitted to AMU who were prescribed an opioid from 1 st to 7 th March 2016. Notes were reviewed to establish: the opioid and dose prescribed; any change to an established opioid or dose on admission; initiation dose of opioid if opioid naïve; any documentation of a rationale behind prescribing in impaired renal function. Laboratory results were reviewed to look for AKI and calculate eGFR.

The audit standards used were the local trust guidelines (4) and the North of England Cancer Network Palliative Care Guidelines (5).

Results 14 patients were prescribed an opioid and only 5 met the audit standards. 4 out of 6 opioid naive patients commenced on morphine IR solution were prescribed a dose higher than recommended. 1 of 5 patients on a long-acting opioid had a correct PRN dose prescribed. A half of patients with a reduced eGFR were prescribed morphine. There was no documentation regarding rationale behind opioid prescribing.

Conclusion The results demonstrated that opioid prescribing on AMU did not adhere to local or regional guidance.

Recommendations

1. Conduct a live audit of patients admitted to AMU over two weeks to expand data

2. Develop specific guidance for opioid adjustment in AKI and for initiating opioids in patients with a reduced eGFR on AMU.

3. Share audit findings and conduct teaching for acute medicine trainees regarding opioid prescribing in acute medical admissions.

4. Repeat audit after interventions taken place.
P-44 THE EFFECTS OF EHEALTH INTERVENTIONS IN PALLIATIVE CARE: A META-REVIEW

${ }^{1}$ Hannah O'Donnell, ${ }^{1}$ Claudia Pagliari, ${ }^{1,2}$ Anne Finucane. ${ }^{1}$ University of Edinburgh; ${ }^{2}$ Marie Curie Hospice Edinburgh

\subsection{6/bmjspcare-2017-00133.44}

Background eHealth involves the use of information and communication technologies (ICTs) for the delivery of healthcare and health information, including direct consumer technologies. eHealth strategies may help alleviate the burden on health systems in a cost-effective way and expand palliative care services.

Aim To systematically identify and synthesise evidence from published systematic reviews on the effects of eHealth interventions in palliative care for patients, caregivers and health professionals.

Methods Systematic reviews focused on eHealth and palliative care were eligible for inclusion in this meta-review. Nine databases including MEDLINE, EMBASE, PsychINFO, and the Grey Literature Report were searched for reviews in any language between 2006 and 2016. The Assessment of Multiple Systematic Reviews (AMSTAR) tool was used to critically appraise all included reviews. Data was then extracted and results were presented in a narrative synthesis.

Results Thirteen reviews were included. Methodological quality was low to moderate with AMSTAR scores ranging from 2 to 5 out of 11 . eHealth interventions were primarily used for facilitating communication, symptom reporting and monitoring, education, information provision, clinical consultations, and decision-making in palliative care settings. There were positive effects of eHealth interventions on cost-effectiveness, decisionmaking, communication, education, and support for patients, caregivers and health care professionals. Inconsistent findings were reported regarding effects on quality of life (QOL), depression, and anxiety.

Conclusion The majority of reviews on eHealth interventions in palliative care report positive effects of interventions on patients, caregivers and professionals. While there were inconsistent findings in regards to depression, anxiety, and QOL, no negative outcomes were reported. This provides promising evidence for the value of eHealth interventions in palliative care. Further research, cost-analyses, and clinical studies are needed to strengthen the evidence base for eHealth interventions and to inform policy in this area.

\section{P-45 AUDIT OF ADULTS WITH INCAPACITY DOCUMENTATION IN AN ACUTE PALLIATIVE CARE UNIT}

Mairi Finlay. NHS Grampian, Aberdeen, UK

\subsection{6/bmjspcare-2017-00133.45}

Background In-patients receiving palliative care may lack capacity to make decisions regarding their medical treatment for many reasons e.g. delirium, dementia. If it is felt that a person cannot consent to treatment an Adults with Incapacity (AWI) Act (Scotland) Section 47 certificate can be completed, allowing healthcare staff to provide treatment while enshrining a number of safeguards for the patient. In 2016 NHS 